\title{
Novel Class of Tertiary Phosphine Ligand Based on a Phospha-adamantane Framework and their Use in the Suzuki Cross-Coupling Reactions of Aryl Halides Under Mild Conditions.
}

George Adjabeng ${ }^{\mathrm{a}}$, Tim Brenstrum ${ }^{\mathrm{a}}$, Jeffrey Wilson ${ }^{\mathrm{a}}$, Christopher Frampton ${ }^{\mathrm{b}}$, Al Robertson ${ }^{\mathrm{c}}$, John Hillhouse $^{c}$, James McNulty ${ }^{\mathrm{a}}$, and Alfredo Capretta ${ }^{\mathrm{a}}$

${ }^{a}$ Institute of Molecular Catalysis, Department of Chemistry, Brock University, St. Catharines, Ontario, L2S 3A1; ' Department of Chemistry, University of Southampton, Highfield, Southampton, SO17 1BJ, U.K; ${ }^{c}$ Cytec Canada Inc., PO Box 240, Niagara Falls, Ontario, L2E 6T4, Canada.

\section{SUPPORTING INFORMATION}

General. Starting materials were purchased from Aldrich Chemical Co or Fluka. Di( $\mu$-acetato)bis[o(di-o-tolylphosphino)benzyl] dipalladium was purchased from Strem. o-Tolyboronic acid, phenylboronic acid, 4-methoxyphenylboronic acid, and 4-acetylphenylboronic acid were recrystallized from water prior to use. All aryl iodides, bromides and chlorides were distilled or recrystallized prior to use, with the exception of 4-chlorobenzonitrile, 2-chloropyrindine, and 4-bromoacetophenone, which were used as received. $\mathrm{K}_{3} \mathrm{PO}_{4}$ and $\mathrm{Cs}_{2} \mathrm{CO}_{3}$ were ground to a fine powder using a mortar and a pestle and dried in a vacuum oven prior to use. Toluene was distilled under argon from sodium. ${ }^{1} \mathrm{H}-{ }^{13} \mathrm{C}$ - and

${ }^{31} \mathrm{P}-\mathrm{NMR}$ spectra were recorded at $300 \mathrm{MHz}, 75 \mathrm{MHz}$ or $81 \mathrm{MHz}$ respectively, on a Bruker Avance DPX-300 spectrophotometer with chloroform-d as the solvent and internal reference unless otherwise noted. MS analysis was performed on a Kratos Concept 1S spectrometer. Ions for low resolution mass spectra (MS) and high resolution mass spectra (HRMS) were generated using electron impact (EI). GC analysis was performed on the HP-6890 with FID. For both structures 1, 2 and 3, unit cell determinations and data collected on a Rigaku AFC7R diffractometer with graphite-monochromated 
Mo-K $\alpha$ radiation $(\lambda=0.71069 \AA)$. The structures were solved with direct methods, SHELXS-90 ${ }^{1}$ and refined with full-matrix least-squares refinement on $F^{2}$ with SHELXL-93. ${ }^{2} R$-values; $R 1=\Sigma|| \mathrm{F}_{\mathrm{o}} \mid-$ $\left|\mathrm{F}_{\mathrm{c}}\right||/ \Sigma| \mathrm{F}_{\mathrm{o}} \mid$, and $w R 2=\left[\Sigma w\left(F_{o}{ }^{2}-F_{c}{ }^{2}\right)^{2} / \Sigma w\left(F_{o}{ }^{2}\right)^{2}\right]^{1 / 2}$. Further details of the crystal structure investigation can be obtained form the Director of the Cambridge Crystallographic Data Centre, University Chemical Laboratory, Cambridge CB2 1EW, U.K.

\section{Experimental Procedures and Compound Characterization.}

1, 3, 5, 7-Tetramethyl-2, 4, 8-Trioxa-6-Phospha-adamantane (1). Prepared according to the procedure described by Epstein and Buckler. ${ }^{3}$ Melting point 88-90 ${ }^{\circ} \mathrm{C}^{3} ;{ }^{1} \mathrm{H}$ NMR $(300 \mathrm{MHz})\left(\mathrm{CDCl}_{3}\right) \delta 1.38,1.39$ (s, $3 \mathrm{H}), 1.44,1.49(\mathrm{~d}, J=5.7 \mathrm{~Hz}, 3 \mathrm{H}), 1.71-1.94(\mathrm{~m}, 4 \mathrm{H}), 3.08(\mathrm{~d}, J=190 \mathrm{~Hz}, 1 \mathrm{H}) ;{ }^{13} \mathrm{C}$ NMR $(75$ $\mathrm{MHz})\left(\mathrm{CDCl}_{3}\right) \delta 27.8,29.0,29.5[\mathrm{~d}, J(\mathrm{P}-\mathrm{C})=13.3 \mathrm{~Hz}], 30.2[\mathrm{~d}, J(\mathrm{P}-\mathrm{C})=22.7 \mathrm{~Hz}], 42.7[\mathrm{~d}, J(\mathrm{P}-\mathrm{C})=$ $14.5 \mathrm{~Hz}], 45.2[\mathrm{~d}, J(\mathrm{P}-\mathrm{C})=4.8 \mathrm{~Hz}], 70.3[\mathrm{~d}, J(\mathrm{P}-\mathrm{C})=3.5 \mathrm{~Hz}], 72.0[\mathrm{~d}, J(\mathrm{P}-\mathrm{C})=18.4 \mathrm{~Hz}], 96.4$, 96.7;

${ }^{31} \mathrm{P}$ NMR (81 MHz) $\left(\mathrm{CDCl}_{3}\right) \quad \delta-49.2 ; \mathrm{MS} \mathrm{m} / z 216\left(\mathrm{M}^{+}, 15 \%\right), 116(30), 101$ (32\%), 69 (24\%), 43 (100). Crystallographic data for $1: \mathrm{C}_{10} \mathrm{H}_{17} \mathrm{O}_{3} \mathrm{P}, \mathrm{MW}=216.21, T=123(1) \mathrm{K}$, monoclinic space group $P 2{ }_{1} / c, a=8.1422(3) \AA, b=8.0756(2) \AA, c=16.6229(5) \AA, \beta=94.155(1)^{\circ}, V=1090.14(6) \AA^{3}, Z=4$, $R_{1}=0.0300[I>2 \sigma(I)], S=1.002$.

1, 3, 5, 7-Tetramethyl-2, 4, 8-Trioxa-6-Phenyl-6-Phospha-adamantane (2). A mixture containing 1,3,5,7-tetramethyl-2,4,8-trioxa-6-phospha-adamantane (1, $100 \mathrm{~g}, 0.46 \mathrm{~mol})$, bromobenzene (75 g, 0.48 mol), triethylamine (45 g, $0.45 \mathrm{~mol})$ and di( $\mu$-acetato)bis[o-(di-o-tolylphosphino)benzyl] dipalladium (II) $(310 \mathrm{mg}, 0.331 \mathrm{mmol})$ was heated to reflux under nitrogen, with magnetic stirring. After 24 hours under these conditions, approximately 79\% conversion of 1 to $\mathbf{2}$ was observed by GC while a 90\% conversion observed after 40 hours. The reaction mixture was washed with water $(200 \mathrm{~mL})$ and the organic layer separated. Evaporation gave the crude product (160g) as a reddish orange solid. Recrystallization of this material with $95 \%$ ethanol $(250 \mathrm{~mL})$ gave a white crystalline solid $(100 \mathrm{~g}, 75 \%$ yield). The compound showed: m.p. $107-110{ }^{\circ} \mathrm{C} ;{ }^{1} \mathrm{H}$ NMR $(300 \mathrm{MHz})\left(\mathrm{CDCl}_{3}\right) \delta 1.25(\mathrm{~d}, J=12.9 \mathrm{~Hz}$, $3 \mathrm{H}), 1.38$ (br.s, $6 \mathrm{H}), 1.52$ (d, $J=12.7 \mathrm{~Hz}, 3 \mathrm{H}), 1.78-2.11$ (m, $3 \mathrm{H}), 7.36-7.38$ (m, $3 \mathrm{H}), 7.80-7.85$ (m, $2 \mathrm{H}) ;{ }^{13} \mathrm{C}$ NMR $(75 \mathrm{MHz})\left(\mathrm{CDCl}_{3}\right) \delta 26.8[\mathrm{~d}, J(\mathrm{P}-\mathrm{C})=11.5 \mathrm{~Hz}], 27.4[\mathrm{~d}, J(\mathrm{P}-\mathrm{C})=22.1 \mathrm{~Hz}], 27.8,28.0$ $(\mathrm{s}), 36.2[\mathrm{~d}, J(\mathrm{P}-\mathrm{C})=1.6 \mathrm{~Hz}], 45.4[\mathrm{~d}, J(\mathrm{P}-\mathrm{C})=17.4 \mathrm{~Hz}], 73.1[\mathrm{~d}, J(\mathrm{P}-\mathrm{C})=7.6 \mathrm{~Hz}], 73.4[\mathrm{~d}, J(\mathrm{P}-\mathrm{C})=$ $21.8 \mathrm{~Hz}$ ], 96.0, $96.8(\mathrm{~s}), 128.3$ [d, $J(\mathrm{P}-\mathrm{C})=7.2 \mathrm{~Hz}], 129.4(\mathrm{~s}), 133.9$ [d, J(P-C) = $26.9 \mathrm{~Hz}], 135.0$ [d, $J(\mathrm{P}-\mathrm{C})=19.7 \mathrm{~Hz}] ;{ }^{31} \mathrm{P}$ NMR $(81 \mathrm{MHz})\left(\mathrm{CDCl}_{3}\right) \delta-23.6$; MS m/z $292\left(\mathrm{M}^{+}, 16 \%\right), 192$ (10), 177 (39), 43 (71); HRMS: Found 292.1244, Calculated 292.3099. Crystallographic data for 2: $\mathrm{C}_{16} \mathrm{H}_{21} \mathrm{O}_{3} \mathrm{P}, \mathrm{MW}=$ 
292.30, $T=123(1) \mathrm{K}$, monoclinic space group $P 2_{1} / n, a=7.8551(6)(3) \AA, b=9.1866(7) \AA, c=$ $20.8860(17) \AA, \beta=91.016(3)^{\circ}, V=1506.9(2) \AA^{3}, Z=4, R_{1}=0.0348[I>2 \sigma(I)], S=1.007$.

1, 3, 5, 7-Tetramethyl-2, 4, 8-Trioxa-6-(2'-Tolyl)-6-Phospha-adamantane (3). A mixture containing 1,3,5,7-tetramethyl-2,4,8-trioxa-6-phospha-adamantane (1, $100 \mathrm{~g}, 0.46 \mathrm{~mol}), o$-bromotoluene (78 g, $0.46 \mathrm{~mol})$, triethylamine (46 g, $0.46 \mathrm{~mol})$ and nickel acetate tetrahydrate $(350 \mathrm{mg}, 1.41 \mathrm{mmol})$ was heated to reflux under nitrogen, with magnetic stirring. During this time the nickel acetate dissolved, generating a reddish brown solution. After 24 hours at reflux, the conversion of $\mathbf{1}$ to $\mathbf{3}$ was estimated at approximately $81 \%$ via GC. The mixture was washed with water $(250 \mathrm{~mL})$, the layers were separated and the organic layer was stripped of volatile components under nitrogen to give a solid residue. This residue was recrystallized from $95 \%$ ethanol $(200 \mathrm{~mL})$ to give a colourless, crystalline solid $(75 \mathrm{~g}, 55 \%$ yield). The compound showed: m.p. $102-104{ }^{\circ} \mathrm{C} ;{ }^{1} \mathrm{H}$ NMR (300 MHz) $\left(\mathrm{CDCl}_{3}\right) \delta 1.28(\mathrm{~d}, J=12.2 \mathrm{~Hz}$, 3 H), 1.43, 1.44 (s, $3 \mathrm{H}), 1.47$ (d, $J=12.9 \mathrm{~Hz}, 3 \mathrm{H}), 1.89-2.15$ (m, $3 \mathrm{H}), 2.61$ (s, $3 \mathrm{H}), 7.18-7.27$ (m, 3 $\mathrm{H}), 8.15-8.18(\mathrm{~m}, 1 \mathrm{H}) ;{ }^{13} \mathrm{C}$ NMR $(75 \mathrm{MHz})\left(\mathrm{CDCl}_{3}\right) \delta 22.0[\mathrm{~d}, J(\mathrm{P}-\mathrm{C})=25.4 \mathrm{~Hz}], 26.6[\mathrm{~d}, J(\mathrm{P}-\mathrm{C})=$ $11.3 \mathrm{~Hz}$ ], 27.8, (s), 27.9 [d, $J(\mathrm{P}-\mathrm{C})=19.9 \mathrm{~Hz}], 28.0(\mathrm{~s}), 36.0(\mathrm{~s}), 46.0$ [d, $J(\mathrm{P}-\mathrm{C})=18.9 \mathrm{~Hz}], 73.4$ [d, $J(\mathrm{P}-\mathrm{C})=23.2 \mathrm{~Hz}], 74.1[\mathrm{~d}, J(\mathrm{P}-\mathrm{C})=8.0 \mathrm{~Hz}], 96.0,96.8(\mathrm{~s}), 125.8,129.3(\mathrm{~s}), 130.6[\mathrm{~d}, J(\mathrm{P}-\mathrm{C})=5.3$ $\mathrm{Hz}], 132.2[\mathrm{~d}, J(\mathrm{P}-\mathrm{C})=28.4 \mathrm{~Hz}], 133.3[\mathrm{~d}, J(\mathrm{P}-\mathrm{C})=3.1 \mathrm{~Hz}], 145.2[\mathrm{~d}, J(\mathrm{P}-\mathrm{C})=28.1 \mathrm{~Hz}] ;{ }^{31} \mathrm{P} \mathrm{NMR}(81$ MHz) $\left(\mathrm{CDCl}_{3}\right) \quad \delta-38.5$; MS m/z $306\left(\mathrm{M}^{+}, 31 \%\right), 206$ (100), 191 (45), 43 (94); HRMS: Found 306.1384, Calculated 306.3365. Crystallographic data for 3: $\mathrm{C}_{17} \mathrm{H}_{23} \mathrm{O}_{3} \mathrm{P}, \mathrm{MW}=306.32, T=123(1) \mathrm{K}$, monoclinic space group $P 2{ }_{1} / n, a=7.4988(6) \AA, b=28.084(2) \AA, c=8.2246(6) \AA, \beta=110.827(2)^{\circ}, V$ $=1618.9(2) \AA^{3}, Z=4, R_{1}=0.0336[I>2 \sigma(I)], S=1.005$.

1, 3, 5, 7-Tetramethyl-2, 4, 8-Trioxa-6-Tetradecyl-6-Phospha-adamantane (4). A stirred solution of 1,3,5,7-tetramethyl-2,4,8-trioxa-6-phospha-adamantane (1, $256 \mathrm{~g}, 1.19 \mathrm{~mol})$ and 1-tetradecene (400 g, $2.02 \mathrm{~mol})$ was heated to $90-93^{\circ} \mathrm{C}$ and treated with $136 \mathrm{~g}$ of a solution containing of Vazo ${ }^{\circledR} 67(10 \mathrm{~g}$, $0.05 \mathrm{~mol})$ in toluene ( $190 \mathrm{~g}$ ) over $3.5 \mathrm{hr}$. Greater than $99 \%$ of 1 was converted as judged by GC. The mixture was washed with water $(250 \mathrm{~mL})$, the layers were separated and the organic layer was stripped of volatile components under vacuum $(5 \mathrm{~mm} \mathrm{Hg}$ ) to give a solid residue ( $406 \mathrm{~g}, 83 \%$ yield). For the purposes of storage, compound 4 was converted to its $\mathrm{HBF}_{4}$ adduct by adding tetrafluoroboronic acid (48 $\mathrm{wt} \%$ aqueous solution, $1.5 \mathrm{~mL}, 11.4 \mathrm{mmol})$ to a solution of $4(500 \mathrm{mg}, 1.71 \mathrm{mmol})$ in dichloromethane $(25 \mathrm{~mL})$. The resulting mixture was stirred for $10 \mathrm{~min}$ at which time the organic layer was then separated from the aqueous layer, dried over sodium sulphate and filtered. Removal of the 
solvent provided the $\mathrm{HBF}_{4}$ adduct of 4 (824 mg, 97\%) as a white solid. Compound 4 showed: m.p. 41 $42{ }^{\circ} \mathrm{C}\left(\mathrm{HBF}_{4}\right.$ adduct $) ;{ }^{1} \mathrm{H}$ NMR $(300 \mathrm{MHz})\left(\mathrm{CDCl}_{3}\right) \delta 0.88(\mathrm{t}, J=6.5 \mathrm{~Hz}, 3 \mathrm{H}), 1.26(\mathrm{~s}, 6 \mathrm{H}), 1.29(\mathrm{~d}, J$ $=2.8 \mathrm{~Hz}, 3 \mathrm{H}), 1.33(\mathrm{~d}, J=3.6 \mathrm{~Hz}, 3 \mathrm{H}), 1.36(\mathrm{~s}, 4 \mathrm{H}), 1.72-1.98(\mathrm{~m}, 2 \mathrm{H}) ;{ }^{13} \mathrm{C} \mathrm{NMR}(75 \mathrm{MHz})$ $\left(\mathrm{CDCl}_{3}\right) \delta 14.1(\mathrm{~s}), 21.1[\mathrm{~d}, J(\mathrm{P}-\mathrm{C})=21.9 \mathrm{~Hz}], 26.8[\mathrm{~d}, J(\mathrm{P}-\mathrm{C})=12.7 \mathrm{~Hz}], 27.8,28.0,28.0[\mathrm{~d}, J(\mathrm{P}-\mathrm{C})=$ $17.2 \mathrm{~Hz}$ ], 28.4 [d, $J(\mathrm{P}-\mathrm{C})=21.7 \mathrm{~Hz}$ ], 29.3, 29.3, 29.5, 29.6, 29.6 (m), 21.4 [d, J(P-C) = 12.5 Hz], 31.9, $31.9(\mathrm{~s}), 44.5[\mathrm{~d}, J(\mathrm{P}-\mathrm{C})=15.0 \mathrm{~Hz}], 72.1[2 \mathrm{~d}, J(\mathrm{P}-\mathrm{C})=22.6 \mathrm{~Hz}], 95.7,96.6(\mathrm{~s}) ;{ }^{31} \mathrm{P} \mathrm{NMR}(81 \mathrm{MHz})$ $\left(\mathrm{CDCl}_{3}\right) \quad \delta-28.0 ; \mathrm{MS} \mathrm{m} / z 412\left(\mathrm{M}^{+}, 5 \%\right), 312$ (56), 269 (18), 130 (44), 115 (100), 43 (97); HRMS: Found 412.3094, Calculated 412.5861.

General Procedure for the Suzuki Cross Coupling Reactions. The arylboronic acid (1 mmol), $\mathrm{K}_{3} \mathrm{PO}_{4}$ or $\mathrm{Cs}_{2} \mathrm{CO}_{3}$ (2 to $\left.2.4 \mathrm{mmol}\right), \mathrm{Pd}_{2}(\mathrm{dba})_{3} . \mathrm{CHCl}_{3}(0.01$ to $0.02 \mathrm{mmol})$, ligand 2 (0.02 to $0.05 \mathrm{mmol}$ added as either a solid or as a $0.20 \mathrm{M}$ solution in toluene) and toluene $(2 \mathrm{~mL})$ are added to the reaction vessel. The reactions are stirred at room temperature (or heated as required) under argon and the course of the reaction monitored by TLC. At the conclusion of the reaction, the reaction mixture is loaded on to small plug of silica gel and washed with copious amounts of $\mathrm{Et}_{2} \mathrm{O}$ or EtOAc. The washings are concentrated and purified by column chromatography on silica gel.

2,2'-Dimethylbiphenyl (Table 1, entry 6). 4-Iodotoluene (218 mg, $1.0 \mathrm{mmol}$ ), o-tolylboronic acid (163 $\mathrm{mg}, 1.2 \mathrm{mmol}) \mathrm{K}_{3} \mathrm{PO}_{4}(509 \mathrm{mg}, 2.4 \mathrm{mmol}), \mathrm{Pd}_{2}(\mathrm{dba})_{3} . \mathrm{CHCl}_{3}(10.4 \mathrm{mg}, 0.0100 \mathrm{mmol})$, ligand 2 (5.8 $\mathrm{mg}, 0.0200 \mathrm{mmol})$, and toluene $(2 \mathrm{~mL})$. After 2.5 hours at room temperature, work up and column chromatography (hexane) yielded $178 \mathrm{mg}$ (98\%) of the title compound as a colorless liquid. ${ }^{1} \mathrm{H}$ NMR $\left(\mathrm{CDCl}_{3}, 300 \mathrm{MHz}\right): \delta 7.34-7.26(\mathrm{~m}, 6 \mathrm{H}), 7.12(\mathrm{~d}, \mathrm{~J}=7.5 \mathrm{~Hz}, 2 \mathrm{H}) .{ }^{13} \mathrm{C} \mathrm{NMR}\left(\mathrm{CDCl}_{3}, 300 \mathrm{MHz}\right)$ : $\delta 142.0,136.2,130.3,129.7,127.6,126.0,20.3 . \mathrm{MS}: 182(\mathrm{M}+, 100 \%), 167(89)$

2-(4-Methoxyphenyl)pyridine (Table 1, entry 15). 2-Chloropyridine (114 mg, $1.0 \mathrm{mmol}$ ), 4methoxyphenylboronic acid (68 mg, $1.1 \mathrm{mmol}), \mathrm{Pd}_{2}(\mathrm{dba})_{3} \cdot \mathrm{CHCl}_{3}(20.7 \mathrm{mg}, 0.020 \mathrm{mmol})$, ligand 2 (0.25 mL, $0.050 \mathrm{mmol}, 0.20 \mathrm{M}$ solution), $\mathrm{Cs}_{2} \mathrm{CO}_{3}$ ( $652 \mathrm{mg}, 2.0 \mathrm{mmol}$ ), and toluene (2 mL). After $24 \mathrm{~h}$ at room temperature, work up and column chromatography (20\% EtOAc in heaxane) afforded a light yellow liquid, $165 \mathrm{mg}(89 \%)$ of the title compound. ${ }^{1} \mathrm{H} \mathrm{NMR}\left(\mathrm{CDCl}_{3}, 300 \mathrm{MHz}\right): \delta 8.44(\mathrm{~d}, \mathrm{~J}=4.5 \mathrm{~Hz}$, 1H), $7.73(\mathrm{~d}, \mathrm{~J}=8.6 \mathrm{~Hz}, 2 \mathrm{H}), 7.52-7.45(\mathrm{~m}, 2 \mathrm{H}), 7.00-7.95(\mathrm{~m}, 1 \mathrm{H}), 7.28(\mathrm{~d}, \mathrm{~J}=8.9,2 \mathrm{H}), 3.64(\mathrm{~s}, 3 \mathrm{H})$. ${ }^{13} \mathrm{C} \mathrm{NMR}\left(\mathrm{CDCl}_{3}, 300 \mathrm{MHz}\right): \delta 160.6,157.1,149.4,137.0,131.8,128.3,121.6,120.1,114.2,55.4$. 
The physical and spectral properties of 4-acetyl-2'-methylbiphenyl ${ }^{4}$ (entries 1, 4, 7 and 11), 4methoxy-2'-methylbiphenyl ${ }^{4}$ (entries 2, 5, 9, 12 and 20), 4-acetyl-4'-methoxybiphenyl ${ }^{4}$ (entries 3, 10 and 17), 4-N,N-dimethylamino-methylbiphenyl ${ }^{4}$ (entry 8), 2-o-tolylpyridine ${ }^{4}$ (entry 14), 2phenyltoluene $^{4}$ (entry 18), 2-phenylpyridine ${ }^{5}$ (entry 13) and 2'methyl-biphenyl-2-carbonitrile ${ }^{5}$ (entry 16) were identical to those previously described.

\section{References.}

(1) Shledrick, G.M. Acta Crystallogr. Sect. A 1990, 46, 467

(2) Shledrick, G.M. SHELXL-93; Program for crystal structure refinement, Gottingen, 1993

(3) Epstein, M.; Buckler, S.A. J. Am. Chem. Soc. 1961, 83, 3279.

(4) Littke, A.F.; Dai, C.; Fu, G.C. J. Am. Chem Soc. 2000, 122, 4020.

(5) Lui, S.-Y.; Choi, M.J.; Fu, G.C. Chem. Commun. 2001, 2408. 